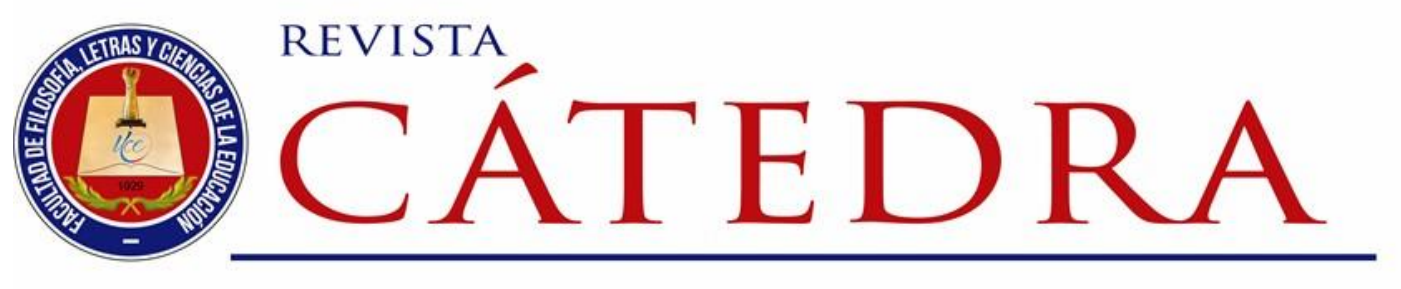

\title{
La cultura como factor de interés para el aprendizaje del francés como idioma extranjero
}

\section{Culture as a factor of interest for learning French as a foreign language}

\author{
Jorge Delgado-Rocha \\ Universidad Central del Ecuador, Quito, Ecuador \\ jgdelgado@uce.edu.ec \\ https://orcid.org/0000-0002-1568-3719 \\ Katherine Sánchez -Medina \\ Universidad Central del Ecuador, Quito, Ecuador \\ kdsanchezm@uce.edu.ec \\ https://orcid.org/0000-0003-2376-5084
}

(Recibido: 13/10/2018; Aceptado: 24/10/2018; Versión final recibida: 03/12/2018)

Cita del artículo: Delgado-Rocha, J. y Sánchez-Medina, K. (2019). La cultura como factor de interés para el aprendizaje del francés como idioma extranjero. Revista Cátedra, 2(1), 27-43.

\section{Resumen}

En este artículo se presenta a la cultura francesa como factor de decisión para los estudiantes de la Alianza Francesa de Quito (AFQ). Elegir al idioma francés para el aprendizaje de un idioma extranjero es fundamental. Se define a la cultura desde un enfoque social, además de determinar las diferentes características que adquiere dependiendo de la sociedad a la que pertenece, de igual forma el objeto de estudio ha sido escogido, debido a que la Alianza Francesa es un referente en la educación del idioma francés como lengua extranjera en Ecuador. La investigación va dirigida a determinar la imagen de la institución que se ha generado gracias a la utilización de la cultura francesa como atrayente para los estudiantes de la AFQ. Se realizó un análisis cualitativo y cuantitativo para observar la importancia que le dan los estudiantes y el directorio de la AFQ a la promulgación de la cultura dentro y fuera de las aulas. Se utilizaron como herramientas de investigación: encuestas sobre la cultura francesa dirigidas a 136 estudiantes de la Alianza Francesa de Quito y, además, una entrevista para conocer la importancia que le da esta institución al uso de la cultura francesa como atrayente educacional. En conclusión, se determinó el nivel de influencia de la cultura de un país para 
aprender su idioma, mediante el uso de diferentes actividades y eventos propuestos e impulsados por la AFQ.

\section{Palabras clave}

Cultura, comunicación, educación, francés, lenguas extranjeras.

\section{Abstract}

In this article, French culture is presented as a decision factor for the students of the Alliance Française of Quito (AFQ) to choose the French language for the learning of a foreign language. It is essential to define the culture from a social perspective, in addition to determining the different characteristics that it acquires depending on the society to which it belongs; thus, the object of study chosen, since the Alliance Française is a reference in the education of the French language as a foreign language in Ecuador. The research is aimed at determining the image of the institution because of the use of French culture as an attraction for the students of the AFQ. A qualitative and quantitative analysis was made to observe the importance that the students and the board of the Alliance Française of Quito give to the promulgation of culture inside and outside the classroom. The research tools used were: surveys on French culture directed to 136 students of the AFQ and, in addition, an interview to know the importance that this institution gives to the use of French culture as an educational attractor. In conclusion, the level of influence of a country's culture to learn its language was determined, using different activities and events proposed and promoted by the AFQ.

\section{Keywords}

Culture, communication, education, French, foreign languages.

\section{Introducción}

El presente trabajo de investigación tiene como objetivo analizar la incorporación de la cultura francesa en las estrategias de la Alianza Francesa de Quito para atraer el interés de los estudiantes a aprender un idioma extranjero. La hipótesis que se maneja en esta investigación es el uso de la cultura como elemento estratégico de comunicación dentro del aprendizaje del francés como idioma extranjero. Además, se analizará el contexto global, pues la sociedad experimenta cambios a través de la denominada globalización. La globalización será vista desde la perspectiva cultural, como un fenómeno de homogenización y unificación de culturas. De esta forma se dará paso a la cultura e interculturalidad y sus implicaciones en la sociedad actual.

A lo largo de los años, las instituciones de enseñanza de idiomas extranjeros han tenido que adaptarse a las nuevas tendencias de la educación que la globalización ha traído consigo y es junto a la cultura que la ampliación y atracción de nuevos tipos de estudiantes ha sido posible. Se escoge entonces a las estrategias de comunicación que utiliza la Alianza Francesa de Quito, como objeto de estudio, debido a que la institución es un referente en la enseñanza y aprendizaje del francés como lengua extranjera tanto en Ecuador. Según la Organización Internacional de la Francofonía, dentro de cincuenta años el francés será la segunda lengua más hablada en el mundo. De igual manera, el francés es el tercer idioma más utilizado en los negocios, lo que vuelve al aprendizaje como punto de gran importancia. 
las diferencias culturales, más que abocar a choques culturales, pueden convertirse en oportunidades para sinergias culturales. Mediante la toma de conciencia de los procesos culturales propios y de los compañeros, estudiantes y profesores pueden reflexionar acerca de sus culturas de aprendizaje, exponer sus dificultades e intentar solventarlas (Trujillo, 2010, pág. 153).

La Alianza Francesa de Quito fue fundada en 1953, convirtiéndose en la primera Alianza Francesa del Ecuador. Desde sus inicios se ha posicionado como un referente en el aprendizaje del francés como lengua extranjera en nuestro país. Dentro de sus instalaciones se realizan actividades culturales en las que se interrelaciona la cultura ecuatoriana y la cultura francesa. Además, la Alianza Francesa es la única institución de aprendizaje de francés que cuenta con diplomas de certificación del idioma avalado por el Ministerio de educación francés, lo que les da una alta ventaja frente a los demás centros de idioma.

Esta investigación se realizará desde el enfoque sistémico, entendido como "una teoría lógicamatemática que se propone formular y derivar aquellos principios generales aplicables a todos los sistemas" (Betarlanffy, 1978, pág. 34). Dentro del enfoque sistémico, vemos a la institución como un sistema, en donde cada una de las partes cumple una función específica, que entiende el contexto y la globalidad del mismo y como afecta a cada una de estas funciones.

Para cumplir esta perspectiva, se abordará a la información desde una entrevista a Charline Lagarde, Coordinadora Pedagógica de la Alianza Francesa de Quito. También, se cumplirá la perspectiva sistémica, con la realización de encuestas con preguntas cerradas, y con una extensión de no más de seis preguntas; a estas las entendemos desde la perspectiva de Amstrong y Kotler

el método más utilizado para recabar datos primarios es la estrategia más adecuada para reunir información descriptiva. La empresa que desea saber cuáles son los conocimientos, actitudes, preferencias o comportamiento de compra de las personas a menudo puede obtener esa información al preguntarles de manera directa (Philip Kotler, 2012, pág. 109).

El artículo está estructurado en: una revisión literaria sobre cultura, interculturalidad y globalización; a continuación se procederá a la aplicación de un análisis doble sobre las estrategias de comunicación que utiliza la Alianza Francesa de Quito y la introducción de la cultura en estas, para lo que será necesario utilizar una entrevista abierta dirigida a la Directora Pedagógica de la Alianza Francesa. Se utilizará una encuesta con preguntas abiertas y cerradas dirigidas a los estudiantes de esta institución. Para la aplicación de estas herramientas de investigación se utilizó la fórmula de muestreo para universos finitos y se obtuvo una muestra de 136 estudiantes que realizan actualmente sus estudios dentro de la Alianza Francesa. Seguidamente se realizó la interpretación de los resultados obtenidos tanto de la entrevista como de las encuestas. Finalmente, con base en los resultados obtenidos se propusieron determinadas conclusiones y recomendaciones sobre la importancia de la cultura francesa como factor atrayente para que estudiantes de la propia Alianza Francesa culminen sus estudios de legua extranjera. 


\section{Revisión de la literatura}

\subsection{Cultura y sus estudios}

El término cultura, desde su raíz latina, era entendido en un principio como el cultivo de la tierra, o como un referente del trabajo, pero después, dio paso a su significado como el cultivo del espíritu. Actualmente, el término cultura es bastante utilizado dentro de la sociedad, pero esta palabra, tiene varias significaciones, desde diferentes perspectivas. Puede ser esa característica intangible de la sociedad que hace que se vuelva única.

Inicialmente la cultura según Bauman (2011), fue considerada como un agente de cambio, que tenía como misión educar a las masas y cambiar sus costumbres. Pero, en la modernidad o como él la llama, modernidad líquida, la cultura ha perdido ese sentido, pues ya no tiene como misión ilustrar e iluminar al pueblo, sino seducir al público. En esta sociedad de consumo, se busca crear necesidades nuevas y a su vez garantizar una permanente insatisfacción.

El concepto francés de "culture" emergió como un nombre colectivo para las fuerzas gubernamentales en pos de fomentar el aprendizaje, suavizar y mejorar los modales, refinar los gustos artísticos y despertar necesidades espirituales que el público no había sentido hasta entonces, o bien no era consciente de que sentía (Bauman, 2011, pág.34).

La modernidad líquida es llamada así por Bauman porque de la misma forma que el líquido, sus etapas en la vida social no pueden mantener su forma por un tiempo largo o prolongado, lo solido se ha diluido, y a diferencia del pasado, estas disoluciones no pueden ser reemplazadas por otras formas sólidas. En nuestra sociedad, el consumo se ha vuelto parte del día a día y en este consumo se encuentra en la cultura.

La cultura de la modernidad líquida se corresponde bien con la libertad individual de elección, y que su función consiste en asegurar que la elección sea y continúe siendo una necesidad y un deber ineludible de la vida, en tanto que la responsabilidad por la elección y sus consecuencias queda donde la ha situado la condición humana de la modernidad líquida: sobre los hombros de los individuos, ahora designado gerente general y único ejecutor de su 'política de vida' (Bauman, 2011, pág.35).

Desde siempre la sociedad tiene el instinto de identificarse a través de ideas, reacciones y pautas de conducta habitual que los miembros han adquirido sea por instrucción, o por imitación. La migración de personas es parte integral de la modernidad, y a su vez de la modernización. Según Bauman (2011) a este fenómeno lo separa en tres fases:

1. Emigración de Europa hacia el resto del mundo: Europa, al ser la región más poblada del mundo en la época, se movilizó hacia las tierras supuestamente vacías. Durante estas migraciones, la invasión y matanza fue justificada con la misión del hombre blanco y su cultivo cultural.

2. Migración hacia un modelo de asimilación: Cuando las colonias estaban por terminar algunas de las poblaciones nativas de los lugares invadidos viajaron hacia los países de sus colonizadores, por lo que al llegar se convirtieron en "minorías étnicas". 
3. La tercera fase, es en la que vivimos actualmente, un proceso que sigue en desarrollo y que, a pesar de los esfuerzos de frenar el acceso de inmigrantes a otros países por parte de sus gobiernos, este proceso continua.

Estos procesos migratorios han dado como resultado muchos más cambios en la historia de la humanidad, incluyendo fenómenos que son estudiados actualmente, uno de estos es la globalización, el fenómeno más estudiado por las ciencias sociales. Existe algo conocido como "choque cultural" que, generalmente sucede con personas que se insertan en otra cultura, distinta a la cultura de origen. Puede darse también cuando un individuo aprende un segundo idioma, en ambos casos, se pueden dar como síntomas, el rechazo, infelicidad, entre otros.

Este estado de estrés llena al estudiante de incomodidad y discrepancias entre su cultura y el nuevo mundo que está explorando; es un sentimiento normal en este tipo de situaciones, pues no se está acostumbrado a la socialización en ese espacio. La forma de superar este choque es dejarse adentrar en la cultura, conociendo gente nativa de ese espacio. Puede definirse entonces, como una situación temporal, pues gracias a la socialización y entendimiento, ese sentimiento de angustia desaparece.

\subsection{La globalización y su efecto en la sociedad}

Para hablar de globalización, es necesario especificar desde que punto de vista se lo va a tomar, puesto que la globalización puede ser analizada desde el sentido de la universalización de la economía, pero también puede verse desde el aspecto cultural e incluso desde lo político. "Este término hace referencia a algo que ocurre en todo el mundo, que afecta a todos los países y a casi toda la población mundial” (Ander-Egg, 2016, pág. 17). A este proceso que afecta a todos, Ander-Egg menciona que existió mucho antes del capitalismo, un aspecto particular del proceso de mundialización/planetarización, que tiene inicios en el siglo $\mathrm{XV}$, pero que es apenas a finales del siglo XX junto a los avances tecnológicos que tiene impactos más profundos. Para las organizaciones, Ander-Egg menciona que la globalización tiene otro significado:

La globalización, para las empresas de mi grupo, es la libertad de invertir cuando y donde quieran, de producir lo que quieran, de comprar y vender donde quieran y de sufrir las menores restricciones posibles derivadas de la legislación liberal y convenciones sociales (Ander-Egg, 2016, pág. 17).

Roche y Oliver (2015) mencionan que la globalización tiene que ver con un proceso de homogenización de la cultura, en donde exista un solo modo de vida, una uniformidad de comportamientos, pensamientos y hasta ocio. Para esto se ha utilizado a los medios de comunicación como fuente de difusión estratégica a nivel global, pues vaciar de significado a las culturas de los otros países, da una ventaja sustancial para convertir a la nueva cultura en mercancía dentro de la mente de las personas que conforman otros países. "Cada avance en la tecnología posibilita, sin duda, la aceleración del proceso, pero no supone cambios en lo fundamental de este: en su lógica, en su sistema de valores, en sus objetivos" (Roche y Narbona, 2005, pág. 69). Por lo tanto, no se puede hablar de interculturalidad, pues mientras en la globalización se interpone la unificación, en la interculturalidad se plantea un diálogo entre culturas de manera equitativa. 


\subsection{La interculturalidad y su rol en la actualidad}

Los estados-nación modernos poseen como característica principal la pluralidad de culturas dentro de su territorio, es decir que, ya no se habla de una sola cultura sino de varias culturas diversas y con sus propias particularidades. La unión de estas culturas da como resultado diversidad y la generación de nuevas diferencias y de nuevos grupos sociales.

Históricamente, la estrategia de los grupos dominantes de los nuevos Estados fue reducir la diversidad interior y promover la homogeneidad cultural mediante el control de la escuela y los medios de masas, la imposición de una lengua determinada, unos símbolos y unos rituales, la delimitación de unas fronteras, la reconstrucción y la invención de la historia, y el hecho de imaginar la existencia de una unidad monolítica y monocultural que contrastaba con el mundo exterior (Beltrán, 2015, pág. 66).

La interculturalidad está ligada a aspectos como la migración, en donde se realiza un proceso de intercambio de puntos de vista abiertos, respetuosos, entre personas de distintos orígenes, tradiciones, lenguas, religiones, entre otros. Sirve para comprender de mejor manera las diversas prácticas y visiones del mundo. A través de la interculturalidad se busca la tolerancia y respeto. Es la capacidad de interrelacionar la cultura de origen con la cultura extranjera. "El intercambio se produce sobre una base de igualdad entendida como la igual aportación de todos los grupos implicados." (Beltrán, 2015, pág. 80). El intercambio es la característica principal de la interculturalidad. La interculturalidad dentro del proceso de educación de los estudiantes tiene un carácter fundamental, ya que la educación debe permitir espacios de absorción e integración entre diferentes culturas. "Cuando se habla de educación intercultural, se deben explorar detalladamente las construcciones discursivas con las que se han dado distintos sentidos al término cultura" (Cortez, 2015, pág. 24).

El idioma como elemento de la cultura es parte integrante de la interculturalidad, por lo tanto, se puede ver reflejada en el Conseil de l'Europe (Consejo de Europa), pues son los creadores del CECRL (Cadre Européen Commun de Reference pour les Langues). Este es un libro de referencia para la enseñanza y aprendizaje de los idiomas que en Europa se hablan (español, francés). Su objetivo principal es la organización del aprendizaje de idiomas y la homologación de diplomas o títulos, la interculturalidad es parte fundamental de este. Dentro de este manual, se ve a quien aprende el nuevo idioma como "apprenant" o aprendiz, lo que le da la categoría de un sujeto o agente social. Por lo tanto, la clase es dirigida a sus necesidades sociales con el entorno. El "apprenant" no aprende dos formas extranjeras de comunicarse, sino más bien, aprende la interculturalidad, una forma de relación entre los idiomas natal y el que es objeto de estudio.

"El objetivo de la interculturalidad es la comunicación, la comprensión de los otros, sin imponerles nuestros valores ni identificarnos necesariamente con los de ellos" (Beltrán, 2015, pág. 81). Dentro de la comunicación, la enseñanza de idiomas junto con la interculturalidad es de suma importancia, pues la cultura del otro tiene elementos que pueden ser considerados sensibles, por ejemplo, en una cultura la forma de saludar de otra puede ser ofensiva o incluso agresiva, entonces la interculturalidad es un aspecto fundamental para el conocimiento y entendimiento del otro. 
El estudiante de una lengua extranjera debe poseer aptitudes y habilidades referentes a la interculturalidad. Estas comprenden la capacidad del estudiante para establecer una relación entre la cultura de origen con la cultura extranjera. Además, es necesario que el estudiante pueda identificar y saber utilizar estrategias de interacción que dependerán del contacto con personas de otra cultura. Finalmente, el estudiante necesita comprender y servir de intermediario entre la cultura de origen y la extranjera, para solucionar malentendidos interculturales, así como también, superar ideas estereotipadas.

La base de la interculturalidad es el entendimiento del otro, la empatía, el intercambio tanto de ideas como de experiencias, mas no la imposición. Está manifestada en la integración armónica de las culturas, de esta forma se evita la pérdida de identidad de los grupos minoritarios ante los grupos dominantes. La integración es la adaptación de todas las partes en igualdad sea esta, de condiciones, de obligaciones, e incluso derechos.

Y aunque esta sea la mejor solución para la adecuada convivencia, no significa que no existan procesos de aculturación o emergencia de nuevas culturas, pues es inevitable. La globalización y la interculturalidad cambian el paradigma de sociedades o grupos homogéneos con una cultura específica dentro de un espacio geográfico único. "La cultura y la identidad se construyen socialmente y se encuentran continuamente en movimiento y en reconstrucción" (Beltrán, 2015, pág. 86).

Es necesario también aclarar la diferencia entre la interculturalidad y la pluriculturalidad. La pluriculturalidad hace referencia al resultado de la interculturalidad, es decir, a las nuevas culturas que han surgido o evolucionado después del contacto, mientras que la interculturalidad, como ya lo definimos, es el dialogo armónico entre estas culturas.

\section{Cultura francesa y la Alianza Francesa de Quito}

La Alianza Francesa es una organización sin fines de lucro, que tiene como objetivo la promoción del idioma francés en los diferentes países del mundo, su sede principal se encuentra ubicada en París, capital de Francia. En 1883, exactamente el 21 julio, se reúnen en el Cercle Saint Simon, intelectuales franceses tales como: el científico Louis Pasteur, el diplomático Ferdinand de Lesseps, los escritores Julio Verne y Ernest Renan y el editor Armand Colin, entre otros. A partir de este momento, se difunde a través del mundo con el mismo fin. Actualmente, la red Alianza Francesa cuenta con 1000 sedes en 136 países. En 2013, la red de Alianza Francesa se vuelve la primera ONG Cultural en el mundo con 6.000 .000 de espectadores y 500.000 estudiantes.

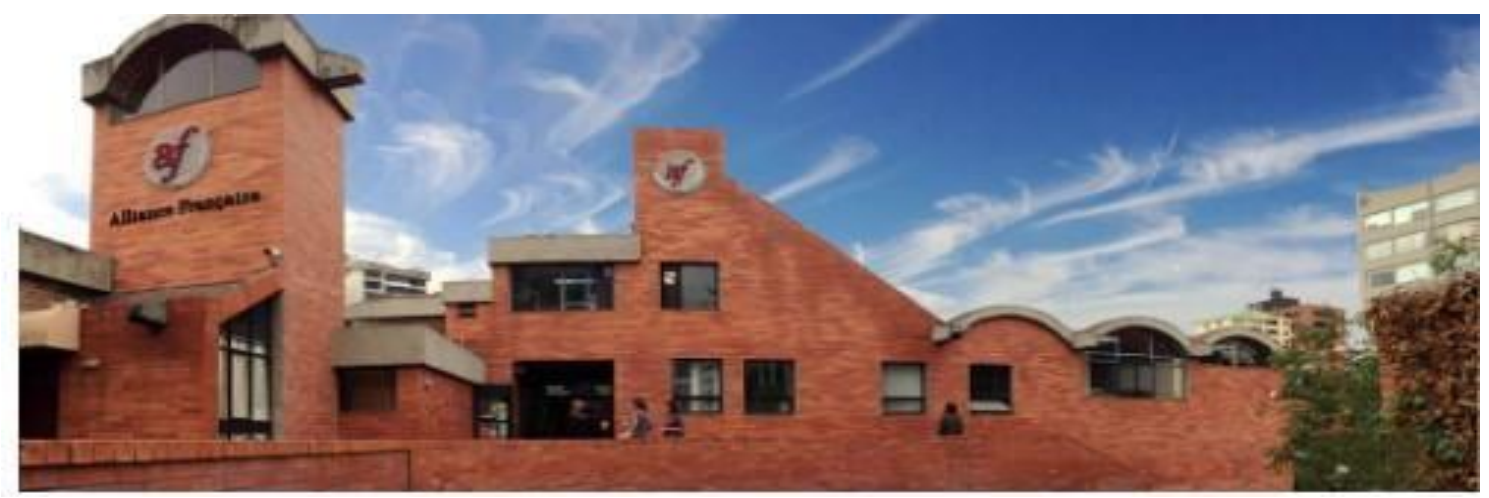

Figura 1. Sede Alianza Francesa de Quito Eloy Alfaro y Bélgica. Fuente: Página Web Campus France equateur.campusfrance.org 
La Alianza Francesa de Quito posee como visión lo siguiente "Alcanzar la excelencia a través de la innovación permanente en la enseñanza del idioma francés y ser un referente de prestigio en la difusión cultural y el vínculo entre las culturas francófona y ecuatoriana" (Alianza Francesa, 2018). Trabaja estrechamente con el servicio de Cooperación y Acción Cultural de la Embajada de Francia para el desarrollo y realización de acciones lingüísticas, artísticas y culturales. La oferta de estudios en la Alianza Francesa de Quito está dirigida al aprendizaje del idioma francés desde su inicio, hasta la preparación y obtención de diplomas certificados por el Ministerio de Educación de Francia, como lo son el TEL, DELF (Diplôme d'études de langue française) A1, A2, B1, B2, y el DALF (Diplôme approfondi de langue française) C1 y C2; estos diplomas sirven para la realización de estudios en el país francófono. "Actualmente, más de 3000 estudiantes y profesores forman parte de una red unida por el idioma y; la cultura francesa y francófona" (Alianza Francesa, 2018). Cabe mencionar también que, dentro de su oferta, se encuentra el programa FLE-VIVIER dirigido a aquellas personas que desean obtener una certificación como profesores de francés.

\section{Objetivos de la investigación}

Considerando el ámbito de análisis de este artículo se determinaron los siguientes objetivos:

1. Definir la importancia de la cultura y la globalización en la sociedad actual, a la hora de decidirse por iniciar y continuar su aprendizaje por un idioma extranjero como es el francés.

2. Analizar las estrategias de comunicación de la Alianza Francesa de Quito, y cómo ha sido integrada la cultura francesa dentro de las mismas.

Para alcanzar los objetivos de esta investigación se realizó un análisis cuanti-cualitativo que fue enfocado a resolver las siguientes interrogantes.

1. ¿Cómo incorpora la Alianza Francesa de Quito la cultura francesa en sus clases y en sus estrategias de captación de estudiantes?

2. ¿Cuál es la importancia e interés que le dan los propios estudiantes de la Alianza Francesa de Quito a la absorción de la cultura francesa?

\section{Método de investigación}

\subsection{Entrevista}

La entrevista tiene como fin la obtención de información de una fuente primaria. "La entrevista no consiste en una charla casual, sino en un diálogo con fines informativos." (López Sobrino y López Cubino, 2012, pág. 11). En el caso de esta investigación las entrevistas a realizarse serán a la Coordinadora Pedagógica Charline Lagarde. Fue el Director General quien designó a Charline Lagarde como la encargada del apoyo de información para esta investigación. Una vez que se realizó las entrevistas, se denotó que la información brindada, en el análisis de la misma, sería duplicada, pues tanto la coordinadora pedagógica como la directora de comunicación tenían la misma información y datos.

\subsection{Encuesta}

Dentro de esta investigación, se utilizará a la encuesta como método de recolección de datos, pues a través de esta, podemos obtener la información de una fuente primaria. Las encuestas pueden ser realizadas personalmente, a través de internet gracias a diversos 
programas creados con este fin y también telefónicamente, en el caso de la presente, las encuestas se realizaron personalmente.

Para empezar, es necesario realizar un cuestionario diseñado para obtener información específica. Las personas que participan de la encuesta han sido previamente seleccionadas a través de la obtención de una muestra representativa.

La investigación por encuesta, el método más utilizado para recabar datos primarios, es la estrategia más adecuada para reunir información descriptiva. La empresa que desea saber cuáles son los conocimientos, actitudes, preferencias o comportamiento de compra de las personas a menudo puede obtener esa información al preguntarles de manera directa (Philip Kotler, 2012, pág. 256).

Para la tabulación de datos, se relacionarán, primeramente, los datos de edad con los datos de sexo, a continuación, será preciso conocer la edad y el nivel de estudios. A partir de la pregunta número uno, se la relacionará con el nivel, pues a través de esta valoración podremos conocer si los estudiantes más avanzados tienen o no más influencia de la cultura.

\subsection{Muestra}

Para la realización de las encuestas, es necesario conocer el número de la población objetivo. La obtención de datos del $100 \%$ de esta población tiene cierto nivel de dificultad, por lo que es necesario obtener una muestra de participantes. Para la obtención de la muestra, se realizó previamente una entrevista a Charline Lagarde, coordinadora pedagógica de la Alianza Francesa de Quito quien supo manifestar que el periodo en el que se realiza esta investigación (agosto 2018- septiembre 2018) es uno de los periodos de estudios con menos frecuencia de estudiantes, debido a la época de vacacionesescolares.

El número de estudiantes inscritos y que asisten regularmente a clase en la Alianza Francesa de Quito durante el año es de alrededor de 5000 personas, y, en el periodo agosto- octubre es de 300 estudiantes. Por lo tanto, a través de la fórmula para la obtención de la muestra en base a un universo finito, dio como resultado 136 alumnos, por lo que se realizó 136 encuestas.

$$
\begin{gathered}
n=\frac{Z^{2} \times N \times p \times q}{e^{2}(N-1)+Z^{2} \times p \times q} \\
n=\frac{1.96^{2} \times 300 \times 0.8 \times 0.2}{0.05^{2}(300-1)+1.96^{2} \times 0.8 \times 0.2}=135.37 \approx 136
\end{gathered}
$$

Ecuación 2

\section{Resultados}

\subsection{Análisis de entrevista}

Para la realización de este artículo, fue importante la información que nos transmitió Charline Lagarde, Coordinadora Pedagógica de la Alianza Francesa. Durante la entrevista se preguntó si el número de estudiantes ha aumentado o disminuido en los últimos años, a lo que Lagarde respondió: “El número de estudiantes no ha aumentado ni disminuido, el 
número es variable, pero de todas maneras considero que se ha mantenido la cantidad." (Lagarde, 2018).

Se puede deducir entonces que, la Alianza Francesa mantiene el número de estudiantes anual, lo que podría ser un indicador de posicionamiento de imagen, tomando un puesto privilegiado frente a otras instituciones de aprendizaje de idiomas. Luego de conocer esta respuesta, fue pertinente conocer la imagen que tienen los miembros de la institución, para lo cual se preguntó, ¿cuál cree que es el motivo de que la AFQ haya tomado este puesto privilegiado sobre los otros institutos de idiomas?

La Alianza Francesa, yo pienso tiene esta posición, con una imagen de prestigio porque siempre intenta, digo intenta, porque nunca es posible hacerlo al 100\%, de mantener una calidad de servicio, una cierta exigencia, y de poder ajustarnos a las necesidades de los estudiantes, es decir, si un estudiante viene, vamos a intentar saber cuáles son sus proyectos, acompañarlo, darle soluciones adaptadas a su perfil, a sus expectativas. Para nosotros cada estudiante es importante, y lo tomamos en consideración (Lagarde, 2018).

Es decir que, el trato personalizado y la adaptación a las necesidades de los estudiantes es la base fundamental para que la Alianza Francesa haya logrado esa posición de prestigio frente a los demás. Por lo tanto, si la Alianza Francesa de Quito, considera que uno de sus fuertes es el servicio al cliente, es también relevante conocer si la cultura francesa es un fuerte atrayente para sus estudiantes. Por lo que se utilizó la pregunta ¿considera que la cultura francesa es un atrayente para que los estudiantes decidan inscribirse?, a lo que Lagarde respondió:

La cultura francesa atrayente para que los estudiantes se inscriban, no estoy segura. Para saberlo, debemos ver con los estudiantes, porque como dije los estudiantes no van necesariamente a asistir a nuestros eventos culturales, yo pienso que más bien, lo que es muy atrayente para los estudiantes al momento de inscribirse son los estudios en Francia. Podría decir que un 50\% de los estudiantes se inscriben realmente por el placer de aprender, entonces quizá pueda estar ligado a la cultura, digamos, cultura general francófona, todo lo que es el conocimiento de una nueva lengua, nueva cultura, gastronomía, viajes, etc. (Lagarde, 2018).

Para Lagarde, que los estudiantes aprendan el idioma francés no está ligado a intereses culturales directamente, pues el 50\% de ellos se inscriben en los cursos por placer, así mismo al no estar interesados en la cultura los eventos culturales que realiza la institución tampoco son de interés. Estos eventos tienen relación tanto a la cultura francesa como a la cultura ecuatoriana, y en ocasiones la capacidad de acogida dentro de las instalaciones es baja por lo que deben trasladarse a un espacio más grande, como es el caso de conciertos.

Entonces, si los estudiantes se inscriben por el placer de aprender, más que por la cultura, necesitamos conocer si los estudiantes completan todos los niveles que ofrece la institución. Con relación a esta pregunta Charline Lagarde dijo lo siguiente: "Generalmente no, no hasta el B2, la mayoría de estudiantes solo siguen hasta el nivel A2, que serían alrededor de 6 periodos. Y en general, cuando deciden continuar hasta el B1 si siguen hasta el B2" (Lagarde, 2018). 
Aunque la AFQ tiene este prestigio y posicionamiento antes mencionado, solamente una parte continúa su aprendizaje hasta el nivel B2, nivel necesario para acceder a una universidad o institución educativa en Francia. Ahora bien, con respecto a las estrategias de comunicación, se consideró pertinente preguntar si existe una diferenciación entre estudiantes y futuros estudiantes. Lagarde dijo entonces que: "Sí, hacemos la diferencia entre quienes ya son estudiantes de la Alianza Francesa y los nuevos estudiantes, para los nuevos tenemos siempre una estrategia de marketing, comunicación diferente" (Lagarde, 2018).

Podemos decir entonces que, la Alianza Francesa de Quito usa targets (nuevos estudiantes y estudiantes inscritos) para sus estrategias de comunicación. Para los estudiantes inscritos se utilizan estrategias de marketing interno referentes a la fidelización, para los nuevos o futuros estudiantes, se utiliza marketing externo como atrayente.

Finalmente, tenemos como conocimiento previo a la entrevista, la realización anual del viaje lingüístico, una estrategia de comunicación de la Alianza Francesa que tiene inmerso el aspecto cultural, debido al contacto directo con la cultura francesa. Entonces, ¿la promoción del viaje lingüístico funciona solo para los estudiantes o pueden personas de fuera también inscribirse?

En general, el viaje lingüístico está abierto a todos los estudiantes de la red pedagógica, una red que constituye alrededor de una decena de colegios en Quito. El nivel mínimo que se pide, es nivel A1. Hemos tenido estudiantes que han querido viajar en el viaje lingüístico, que se han inscrito solo para pasar el nivel A1 y viajar (Lagarde, 2018).

Con la respuesta brindada a esta pregunta, podemos concluir que la cultura francesa si puede servir como un factor de interés para que los estudiantes se inscriban en los cursos de aprendizaje de idioma francés. Tendrán la oportunidad de conocer de forma directa la cultura e idioma francés, aprender sus costumbres y acceder a una sociedad diferente, todo esto mediante un viaje a Francia por apoyo de la AFQ. Este interés nace de los estudiantes debido a que el viaje no es al inicio del curso sino al final, lo que proporciona una oportunidad para la Alianza Francesa de presentar de forma atractiva a todo lo que van a ver los estudiantes durante su viaje. Con el fin de que este viaje sirva como refuerzo para que los mismos estudiantes que iniciaron su curso y viajaron a Francia, sean también los mismos que finalicen sus estudios de francés dentro de la institución.

\subsection{Análisis de encuestas}

La encuesta fue aplicada a 136 estudiantes de un total de 300, de la Alianza Francesa de Quito, del período número 5, es decir, agosto - octubre de 2018. Cabe mencionar que los participantes lo hicieron de forma voluntaria. La encuesta estaba programada para una duración de 5 minutos, pero los participantes tomaron entre 10 y 15 minutos debido a las preguntas en las que debían emitir su opinión.

Al finalizar la encuesta, se generó charla entre los estudiantes sobre estas preguntas. A continuación, vemos la tabulación e interpretación de los resultados de las preguntas realizadas en la encuesta dirigida a los estudiantes de la Alianza Francesa de Quito. 


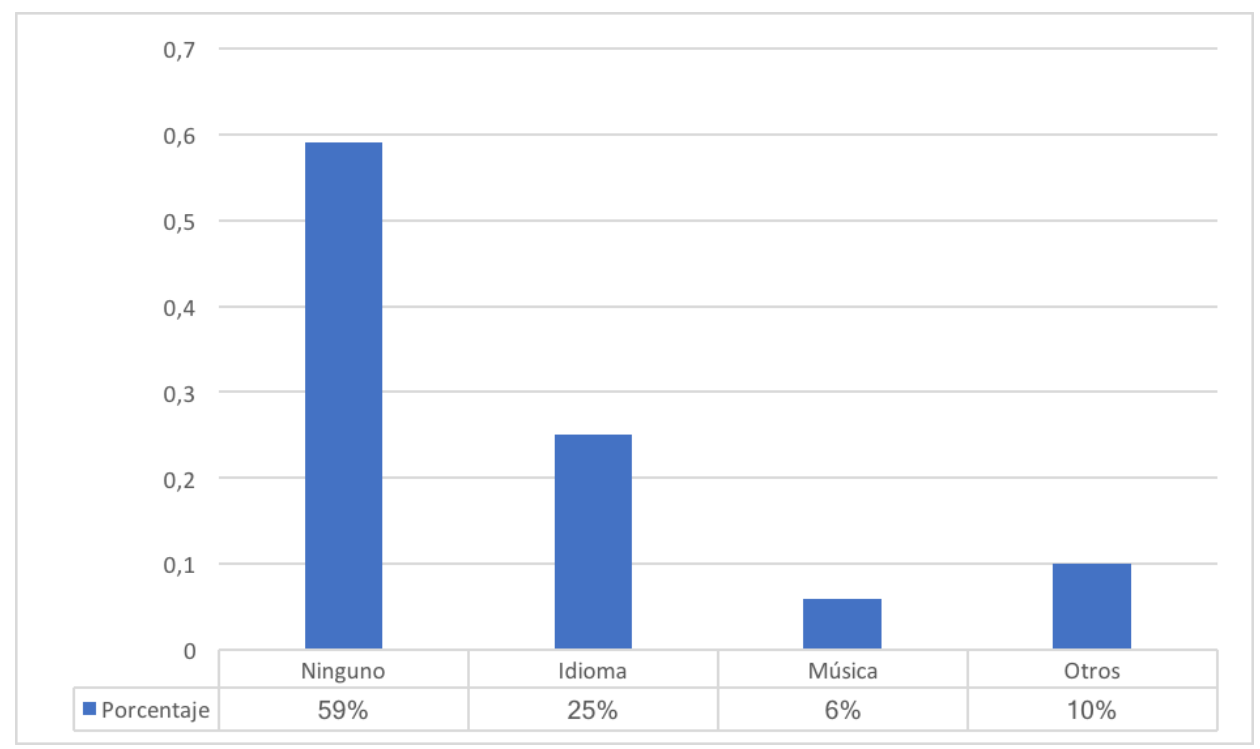

Figura 2. ¿Qué aspectos de la cultura francesa se han impregnado en su día a día gracias a la Alianza Francesa?

Esta pregunta, fue una de las que causó dificultad en los estudiantes a la hora de responder, pues al leerla, tomaban un tiempo para responderla y en ocasiones la saltaban para continuar con el resto de las preguntas para responderla al final. De igual forma, al momento de finalizar la encuesta, los estudiantes generaban un diálogo entre ellos para debatir la respuesta. El 59\% de los encuestados dijo que ningún aspecto de la cultura francesa se ha impregnado en su día a día, el 25\% dijo que el idioma se ha impregnado, el $6 \%$ dijo que la música y el 10\% restante respondió distintos aspectos. Entre las respuestas más destacadas tenemos las siguientes, que han sido transcritas tal cual fueron escritas en la encuesta:

1. La música, conocer su historia (historia de Francia) día a día,

2. La democracia del país, respeto a los derechos humanos,

3. Un poco de la cultura y la historia de Francia,

4. Escuchar música variada, cambiar películas a francés,

5. Festividades, leyes, que se estudian en el libro,

6. La estructura, la educación, la política, las películas, la pintura, los museos son los mejores que he conocido,

7. Mentalidad abierta, cine, música, cocina, moda, multiculturalidad,

8. Cultura francesa en ropa, lenguaje francés, exposiciones de jóvenes, cultura de la comida,

9. Puntos de vista y análisis de problemas. Apreciación por el arte. Aprender a valorar la cultura a la que pertenezco,

10. Varios de los aspectos que se han habituado en mi día a día de la Alianza Francesa y su cultura son más que todo la educación, la forma formal de hablar, la comida, la variedad que hay en cada plato,

11. Juegos de mesa, cine francés y de países francófonos,

12. Las canciones y el entendimiento de algunas palabras francesas que se usan en español, 


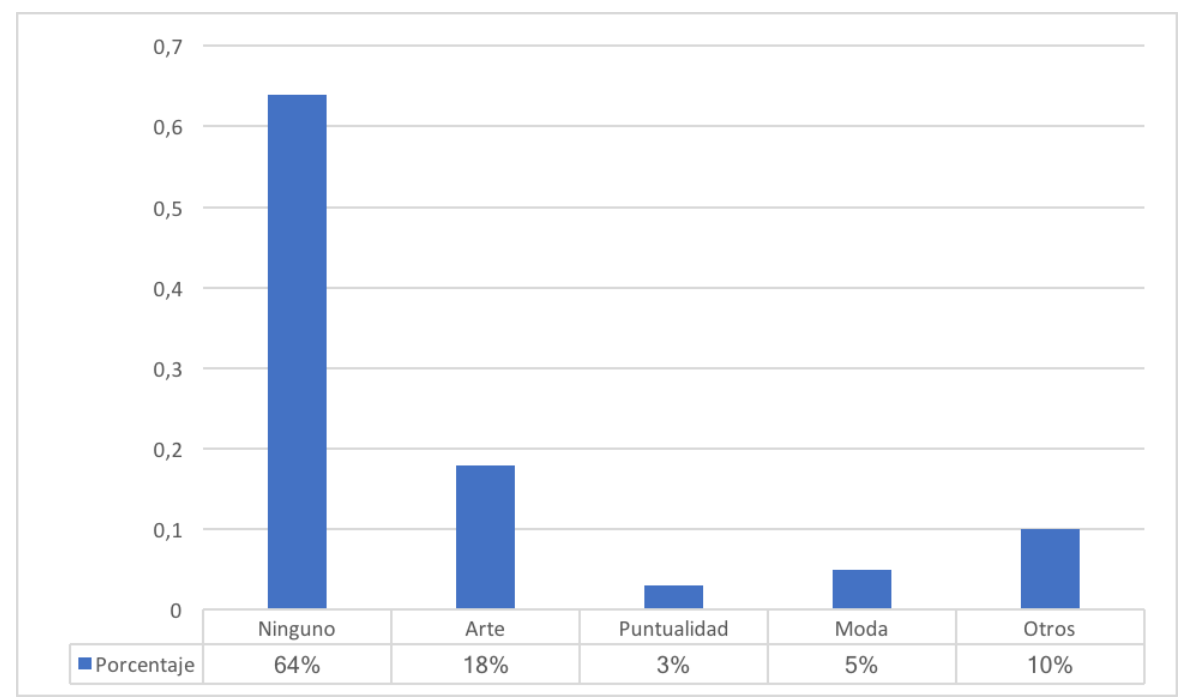

Figura 3. ¿Qué aspectos de la cultura francesa cree que se comparan a aspectos de la cultura ecuatoriana?

Para esta pregunta, los estudiantes tomaron un tiempo para responderla, en ocasiones se generó dialogo entre ellos, así como también fue dejada para ser respondida al final. El 64\% de los encuestados respondió que ningún aspecto es comparable, el 18\% respondió el arte, el 5\% respondió que la moda, el 3\% respondió la puntualidad, el 10\% restante respondió distintos temas como la cordialidad, la educación, la comida, entre otros. A continuación, se copian las respuestas más relevantes de forma textual:

1. Cordialidad de la gente,

2. Son culturas muy diferentes por ejemplo se puede comparar la gastronomía, la moda, la educación. Como aspectos completamenteantónimos,

3. La arquitectura, el arte,

4. La moda, la gastronomía,

5. Las costumbres que se transmiten de generación en generación,

6. Educación, puntualidad, respeto, silencio,

7. Depende de la región, en Quito las personas son más conservadoras que en general los franceses. Sin embargo, el interés por el arte y la comida se parecen mucho,

8. En la cultura francesa hay más educación que en la ecuatoriana,

9. Lastimosamente, no muchos. Las diferencias son demasiado pronunciadas,

10. El saludo habitual entre amigos, el respeto de la comida en familia, la variedad de la comida típica, los museos, y monumentos que conforman al país,

11. En un aspecto negativo que Francia es más organizada que Ecuador y algo positivo para los estudiantes es que las dos lenguas se parecen mucho,

12. Yo pienso que se comparan actualmente en la seriedad y manera de no ser gentiles con los extranjeros, 


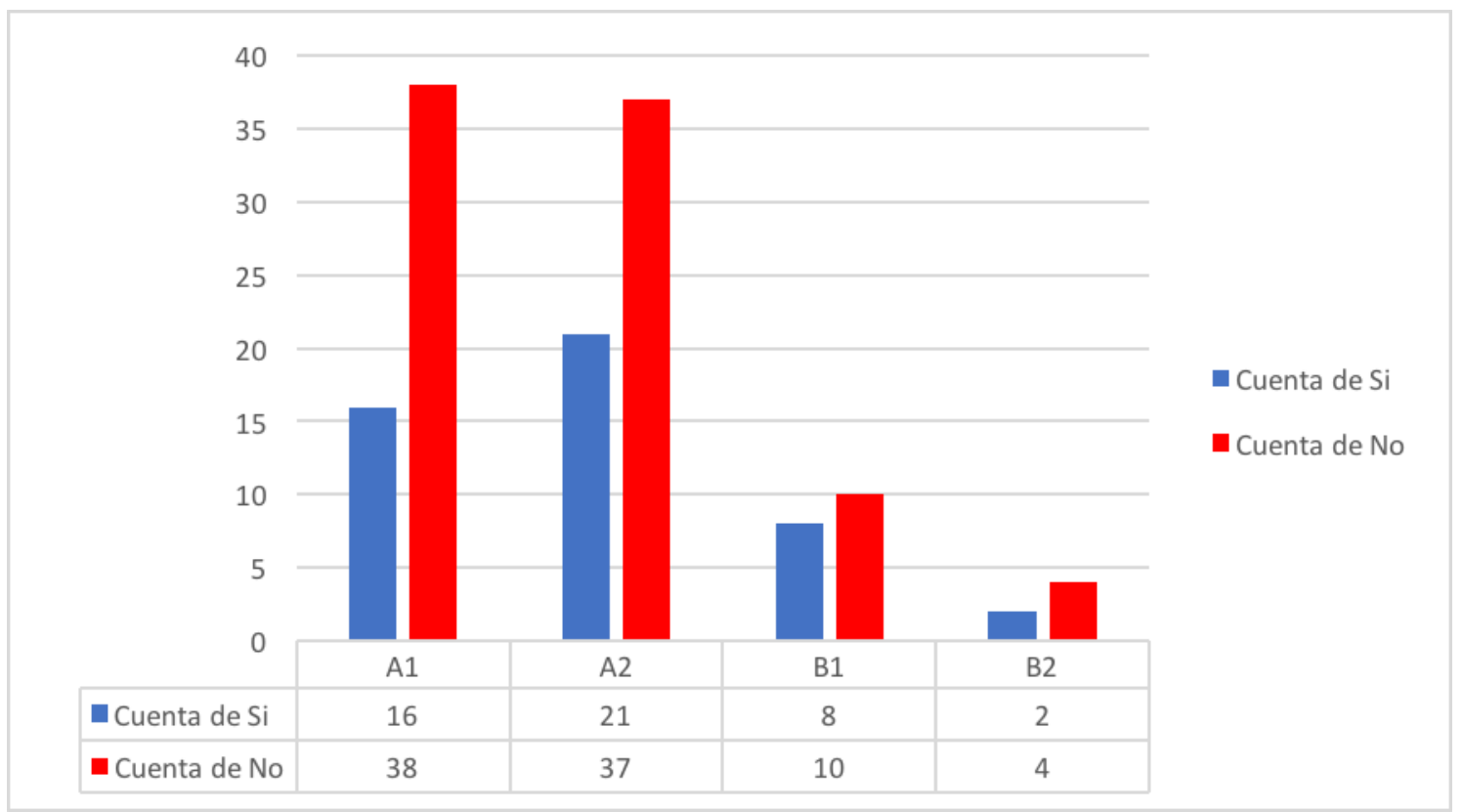

Figura 4. ¿Usted ha asistido a los diferentes eventos culturales realizados por la Alianza Francesa?

Para conocer si los estudiantes participan de los eventos culturales de la Alianza Francesa de Quito, se realizó esta pregunta. El 64,9\% de los participantes no asiste a los eventos culturales de la Alianza, de los cuales 38 personas pertenecen al nivel A1; 37 personas al nivel A2; 10 al nivel B1 y 4 al nivel B2. El 35\% si asiste a los diferentes eventos culturales, esto representa a 16 personas del nivel A1, 21 personas del nivel A2, 8 del nivel B1 y 2 del nivel B2. Para quienes participan de los eventos culturales, se complementó con la pregunta ¿cuáles? Lo que arrojó los siguientes resultados.

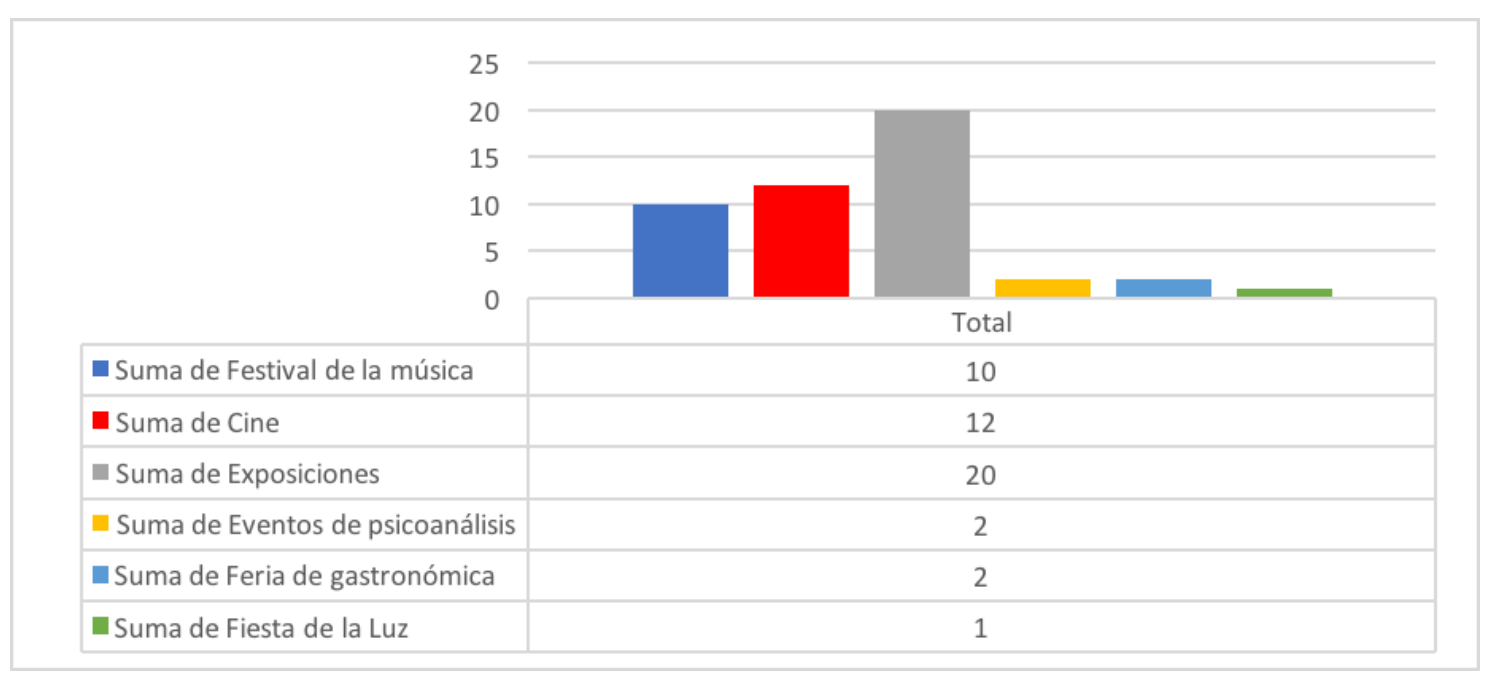

Figura 5. Eventos culturales.

De las 47 personas que asisten a los eventos culturales de la Alianza Francesa, el 42,5\% ha asistido a las exposiciones y galerías de arte, siendo este el evento con más acogida entre los participantes. A continuación, en la imagen 7 , los eventos de cine francés, con un total de $25,5 \%$; le sigue los festivales de música con el $21,2 \%$; los eventos de psicoanálisis y feria gastronómica le sigue con el 4,2\% cada uno y finalmente, la Fiesta de la luz con el $2 \%$. Sobre la Fiesta de la luz, cabe recalcar que no es un evento organizado por la Alianza Francesa, 
pero si tiene participación de la cultura francesa al ser realizado en conjunto por los Municipios de Quito y de Lyon.

\begin{tabular}{|l|l|l|l|l|}
\hline \multicolumn{1}{|c|}{} & \multicolumn{1}{|c|}{5} & & & \\
\hline
\end{tabular}

Figura 6. ¿Considera que los eventos culturales ayudan en el aprendizaje del francés?

En esta pregunta, 6 personas no respondieron, lo que representa el 4,4\% del total de participantes. El 66,9\% piensa que los eventos culturales si ayudan considerablemente en el aprendizaje del francés y el 27,9\% considera que ayuda, pero parcialmente o menos.

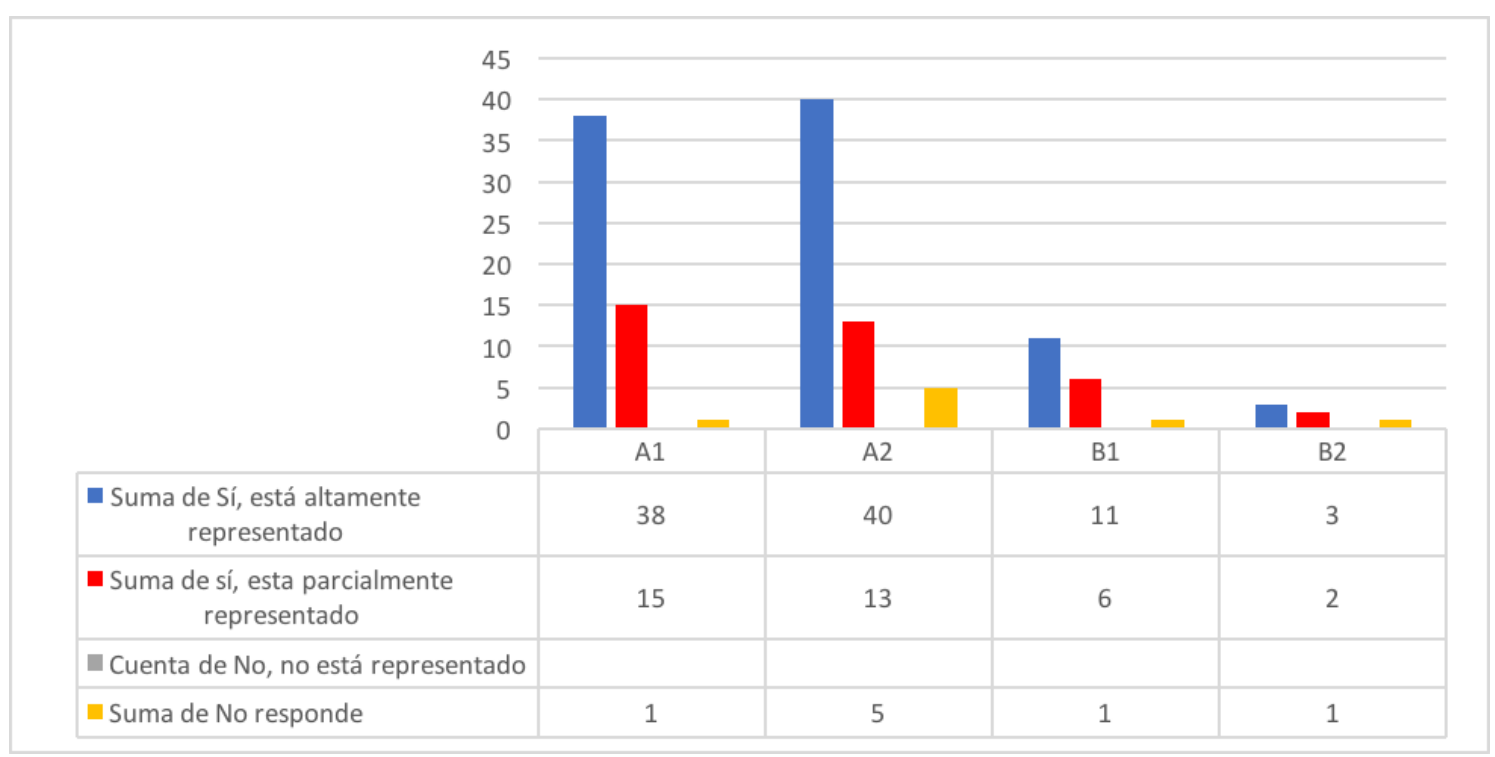

Figura 7. ¿Considera usted que la cultura francesa está bien representada en las estrategias y eventos realizados por la Alianza Francesa?

Una vez más, en esta pregunta 8 personas no respondieron, lo que representa el 5,8\% de los participantes. El 67,6\% considera que la cultura francesa está altamente representada en las estrategias y eventos de la Alianza Francesa, lo que nos refleja que la alianza tiene un buen posicionamiento. El 26,4\% considera que está parcialmente representado.

\section{Discusión y conclusiones}

La cultura tiene varias definiciones según la perspectiva desde la que se la vea, en el caso de esta investigación la hemos tratado desde el aspecto social. Con las migraciones, una 
característica principal de los seres humanos, la cultura se ha ido desarrollando e intercambiando ideas y visiones del mundo, lo que ha dado como resultado a la interculturalidad.

La globalización empezó a ser utilizado en los años ochenta, después de la Segunda Guerra Mundial y la aparición de las nuevas tecnologías de la comunicación y la información. Lo que busca es homogeneizar y unificar a la cultura. En la actualidad este proceso se ve con mayor fuerza, debido a la interrupción de las TIC dentro de la vida cotidiana de las personas, las cuales pueden conocer y observar con mayor facilidad cómo es y de qué forma viven otras personas inmersas en otra sociedad.

La interculturalidad es distinta a la pluriculturalidad, pues la pluriculturalidad hace referencia al resultado de la mezcla de culturas, mientras que la interculturalidad es un dialogo entre estas. El proceso de la interculturalidad ayuda a que las personas dentro de una sociedad delimitada con sus aspectos y características predefinidas puedan acceder a información y conocimientos ajenos a esta sociedad. De esta forma adquieren riqueza de conocimientos y por conclusión un pensamiento más amplio y cosmopolita.

Según las autoridades de la Alianza Francesa de Quito, la cultura no es un atrayente para los posibles estudiantes de la $\mathrm{AFQ}$, sino que más bien el papel que cumple dentro de las estrategias de comunicación es el de fidelizar a los estudiantes para que continúen estudiando. Esto puede ser visto como una oportunidad perdida, ya que cuando se realizó la encuesta dirigida a los estudiantes de la Alianza Francesa de Quito, El 66,9\% de los 136 estudiantes piensa que los eventos culturales si ayudan considerablemente en el aprendizaje del francés. Y el 67,6\% considera que la cultura francesa está altamente representada en las estrategias y eventos de la Alianza Francesa, lo que nos refleja que la AFQ tiene un buen nivel de posicionamiento frente al interés de los estudiantes a la hora de elegir la institución e idioma para empezar su aprendizaje.

Aunque, los resultados de la presente investigación no son concluyentes, es claro que la cultura tiene un gran potencial de ser un factor determinante para los estudiantes a la hora de elegir un idioma a estudiar. Prueba de esto es que el público que asiste a los eventos culturales de la Alianza Francesa de Quito no necesariamente es el mismo público que toma clases en la AFQ. Quiere decir que los posibles estudiantes que son atraídos por los eventos culturales que muestran diferentes aspectos de la cultura francesa y sobre todo la posibilidad de acceder a este nuevo conocimiento y realidad debido a la forma en que muestran la cercanía entre la cultura francesa y ecuatoriana. 


\section{Bibliografía}

Alianza Francesa. (23 de agosto de 2018). Obtenido de Alianza Francesa de Quito: www.alianzafrancesa.org.ec

Ander-Egg, E. (2016). Globalización: El proceso en el que estamos metidos. Córdoba: Brujas.

Bauman, Z. (2011). La cultura en el mundo de la modernidad líquida. (L. Bauman, Trad.) Argentina: Fondo de cultura económica.

Beltrán, J. (2015). La interculturalidad. Barcelona: UOC.

Betarlanffy, L. v. (1978). Teoría General de Sistemas.

Cortes, N. (2015). Interculturalidad, diferencia y etnoeducación: la educación como lugar antropológico. Medellín, Colombia.

Lagarde, C. (7 de septiembre de 2018). La cultura en las estrategias de comunicación de la AFQ. (K. Sánchez, Entrevistador)

López Sobrino, B., y López Cubino, R. (2012). La entrevista y la crónica. Proyectos Mediascopio Prensa. La lectura de la prensa escrita en el aula. Madrid.

Muriel, B. (2015). Momentos en la Alianza Francesa... Quito: Alianza Francesa de Quito.

Philip Kotler, G. A. (2012). Marketing (Decimocuarta ed.). México: Pearson Educación.

Roche Cárcel, J., \& Oliver Narbona, M. (2005). Cultura y globalización. Entre el conflicto y el diálogo. Alicante.

Trujillo, F. (2010). Cultura, comunicación y lenguaje : reflexiones para la enseñanza de la lengua en contexto multiculturales. Barcelona, España

\section{Autores}

JORGE DELGADO-ROCHA como parte de su formación académica, realizó su educación universitaria completa en Ucrania, en la ciudad de Vinnytsia, en la Universidad Técnica Nacional de Vinnytsia, graduado como Ingeniero de Administración de Empresas especializado en Relaciones Internacionales en 2013. Ya en 2015 obtuvo su maestría en Administración de Empresas especializado en Relaciones Internacionales.

Con más de 3 años de experiencia en docencia universitaria en diferentes instituciones, y 3 años de experiencia como traductor de ruso independiente. Actualmente trabaja como docente de la Facultad de Comunicación Social en la Universidad Central del Ecuador. Sus principales temas de investigación incluyen la motivación laboral, la investigación sobre la cultura a niveles internacionales y organizacionales, manejo del e-goberment y gestión de marcas.

KATHERINE SÁNCHEZ-MEDINA como parte de su formación académica, realizó su educación universitaria en Quito, Ecuador, en la Universidad Central del Ecuador, en la Facultad de Comunicación Social, graduada como Licenciada en Comunicación Social con énfasis en Comunicación Organizacional en 2018.

Entre el año 2017 y 2018, realiza los talleres de formación de FLE (Français Langue Étrangère) en el programa "Vivier de professeurs de Français Langue Étrangère", auspiciado por Embajada de Francia en Ecuador y realizado por la Alianza Francesa de Quito. 